INVESTIGACIONES

\title{
Validez consecuencial del programa de asignación de excelencia pedagógica en Chile*
}

\author{
Consequential validity of the teaching excellence certification program in Chile
}

Validade consequente do programa de atribuição de excelência pedagógica no Chile

\author{
Carolina Araya ${ }^{1}$, Sandy Taut ${ }^{2}$, Verónica Santelices ${ }^{2}$, \\ Jorge Manzi ${ }^{2}$ \\ ${ }^{1}$ Escuela de Psicología, Universidad de los Andes. \\ Dirección postal completa: Av. San Carlos de Apoquindo 2200, Las Condes, \\ Santiago de Chile. Teléfono: (562) 4129214. E-mail: caraya@uandes.cl \\ ${ }^{2}$ Centro de Medición, Pontificia Universidad Católica de Chile.
}

\begin{abstract}
RESUMEN
Se presenta el resultado de una investigación realizada entre 2008 y 2010 con docentes y directivos de 30 establecimientos educacionales de dependencia municipal y particular subvencionada en la Región Metropolitana. Empleando metodologías cualitativas, el objetivo del estudio era evaluar la validez consecuencial del programa Asignación de Excelencia Pedagógica, vale decir, verificar empíricamente la Teoría de Acción subyacente e identificar efectos emergentes.

Los principales resultados revelan que los acreditados experimentan mayor autoestima profesional, pero se sienten poco reconocidos por sus pares, directivos y sostenedores. La falta de reconocimientos de la comunidad local influiría en que a nivel de establecimiento la expectativa de diseminar las buenas prácticas de los acreditados no se cumpla. El recurso docente certificado es pobremente utilizado en la gestión pedagógica que realizan los directores y sostenedores.

En las conclusiones se discuten algunos hallazgos y se formulan recomendaciones para favorecer los efectos intencionados por el programa.
\end{abstract}

Palabras clave: certificación de excelencia pedagógica, evaluación docente, evaluación de competencias en profesores, validez consecuencial.

\begin{abstract}
The goal of this qualitative study was to assess the consequences of the "Excellence in Teaching Program", open for teachers of both public and private-subsidized schools since 2002. We interviewed $\mathrm{N}=60$ school leaders from $\mathrm{N}=30$ schools; conducted $\mathrm{N}=5$ focus groups with 35 certified teachers; and interviewed 10 teachers who failed in their application. The main results show that accredited teachers have strengthened their professional self-esteem, and they welcome the monetary incentive; although the burocratic obstacles to receive it lower its value. Social recognition happens more from external sources than the local school community. This could also explain why AEP-certified teachers' best practices do not get disseminated within the local school community. Certified teachers are not seen as a human resource of value for the pedagogical management of the school. Within the conclusions we discuss some of the findings and make recommendations to further the intended program purposes.
\end{abstract}

Keywords: teaching excellence certification, teacher evaluation, teacher competency testing, consequential validity.

* Los autores agradecen al Fondo Nacional de Desarrollo Científico y Tecnológico de Chile (Fondecyt) por el financiamiento del estudio del cual forma parte este artículo ( $\left.\mathrm{N}^{\circ} 1080135\right)$. 


\section{RESUMO}

Apresentam-se os resultados da pesquisa realizada entre 2008 e 2010 com 30 professores e diretores de estabelecimentos de ensino privado subsidiado e municipal na região metropolitana. Utilizando-se metodologias qualitativas, o objetivo foi avaliar a validade consequente do programa de Atribuição de Excelência Pedagógica, ou seja, verificar empiricamente a Teoria de Ação subjacente e identificar os efeitos emergentes. Os principais resultados mostram que os mutuários têm maior autoestima, mas se sentem pouco reconhecidos por seus pares, dirigentes e mantenedores. A falta de reconhecimento da comunidade local pode influeciar no nível de estabelecimento e na expectativa da divulgação de que boas práticas de mutuários não se concretizem. O recurso de professor certificado é pouco utilizado na gestão da educação por diretores e mantenedores. Nas conclusões, discutem-se alguns achados de pesquisa e fazem-se recomendações para promover o alcance dos objetivos pretendidos pelo programa.

Palavras chave: certificação de excelência educacional, avaliação de professores, avaliação de competências de professores, validade consequente.

\section{ANTECEDENTES}

La Asignación de Excelencia Pedagógica (AEP) es un programa que busca acreditar las competencias de los docentes y sus conocimientos de la didáctica y disciplina que enseñan. Se implementa en Chile desde el 2002 y pueden postular -voluntariamente- los docentes de aula que se desempeñan en establecimientos financiados por el Estado o en instituciones privadas que reciben subvención estatal.

Esta iniciativa se enmarca en las políticas públicas orientadas al fortalecimiento de la profesión docente que se comenzaron a implementar a mediados de los noventa. Destaca como una de las primeras iniciativas que introducen un cambio en la estructura salarial de los profesores, ligando los incrementos de sueldo a la calidad del desempeño (Mineduc, 2004 ${ }^{\mathrm{a}}$, Cox, 2003, Mizala \& Romaguera, 2002, Odden \& Kelley, 2002, Harvey-Beavis, 2003; Goldhaber, 2009, Scaflani, 2010). Asimismo, forma parte de las estrategias diseñadas para atraer y retener a profesores competentes, ampliando sus oportunidades de desarrollo profesional (Vaillant, 2009; McKinsey, 2007).

Es un esquema parecido al implementado por el National Board for Professional Teaching Standards (NBPTS) en EE.UU. (Avalos \& Assael, 2006; National Research Council, 2008); los profesores que quieran acreditarse deben rendir una prueba escrita que mide conocimientos disciplinarios y pedagógicos. Además, deben entregar un portafolio que recoge evidencias concretas del proceso de enseñanza-aprendizaje. Ambos instrumentos están basados en las referencias curriculares vigentes y en el Marco para la Buena Enseñanza, que describe los estándares de desempeño profesional docente (Mineduc, 2004b).

Los docentes que obtienen la acreditación son reconocidos públicamente en una ceremonia a la que asisten las autoridades educativas locales. Adicionalmente, reciben un incentivo económico equivalente a un decimotercer sueldo anual(U\$D 1.000 aprox.) por un periodo de 10 años. Para mantener la asignación, los acreditados deben ejercer como docentes de aula en establecimientos que reciben subvención estatal (Mineduc, 2002).

\subsection{VALIDEZ CONSECUENCIAL}

Como todo sistema de evaluación que tiene impactos importantes en la población evaluada (high-stakes testing programs), resulta necesario contar con evidencia que señale 
que los usos e interpretaciones que se hacen de los puntajes contribuyen a la efectividad del sistema escolar. Al mismo tiempo, es importante verificar que no se están produciendo consecuencias adversas imprevistas.

Los documentos normativos relacionados con la medición y evaluación de sujetos destacan que continuamente deben realizarse esfuerzos en materia de validación de estos programas. Este requerimiento forma parte de la responsabilidad que los encargados de los programas tienen con quienes son objeto de evaluación y con aquellos que utilizan sus resultados para la toma de decisiones con consecuencias (Joint Committee on Educational Evaluation, 1988; AERA, APA \& NCME, 1999).

En lo que respecta a AEP, se ha iniciado una línea de investigación sobre la validez de constructo del programa (Manzi, Araya, González, Barros, Bravo, Peirano \& Torres, 2007), pero falta implementar estudios que examinen sus consecuencias. Investigaciones realizadas en otros contextos educativos han mostrado que los programas de evaluación pueden tener sólida evidencia de validez de constructo y sin embargo no logran cumplir con las demandas más complejas de validez consecuencial (Kane, 2002).

El estudio de las consecuencias juega un rol importante en el marco propuesto por el "Joint Study Group on Validity in Accountability Systems" (Fast \& Hebbler, con Joint Study Group on Validity in Accountability Systems, 2004). Estos investigadores destacan que los estudios que den cuenta de la validez consecuencial de los sistemas de evaluación deberían responder dos preguntas fundamentales: (1) ¿Los programas de evaluación están siendo implementados tal como se planificó? (2) ¿Tienen los efectos previstos? ¿Existen efectos no previstos? (Op. cit, 2004). Tales preguntas sólo pueden ser investigadas con precisión si los investigadores y creadores de las políticas se comprometen en un proceso de definición de los antecedentes o la teoría de la acción subyacente al sistema de evaluación (ver también Lane \& Stone, 2002; Baker \& Linn, 2002).

Pese a que existe un debate respecto a si las consecuencias de las mediciones pueden ser incorporadas en la noción de validez (ver Messick, 1992; Linn, 1994, 1997; Shepard, 1997; Lane, Park y Stone, 1998; Messick, 1998; Moss, 1998; Lane y Stone, 2002; Popham, 1997; Mehrens, 1997), todos los autores concuerdan en que las consecuencias de los sistemas de evaluación son importantes como objeto de la investigación educacional.

\section{2. ¿QUÉ EFECTOS SE ESPERABA PRODUCIR CON AEP?}

Para examinar si las consecuencias observadas de AEP coinciden con lo que creadores del programa intentaron provocar, y para identificar consecuencias que no estaban entre las previstas por los impulsores del programa, fue necesario reconstruir -en primer término- la Teoría de Acción de AEP. Según Patton (2008), la Teoría de Acción de un programa "es la articulación sencilla de una cadena de procesos de la que se esperan ciertos resultados. Los nexos entre los procesos y los resultados se hacen explícitos" (p. 223).

De esta forma, entre 2007 y 2008 fueron entrevistados once representantes de cuatro grupos de interés implicados en el diseño e implementación del programa, esto es, Ministerio de Educación, Ministerio de Hacienda, Colegio de Profesores y centros universitarios encargados de la implementación de AEP. Con esto se pretendía describir el modelo lógico o teoría de acción que explica cómo se articulan los componentes del programa para producir los efectos esperados, en opinión de sus creadores. 
Los resultados de este primer estudio revelaron un modelo subyacente general, con vacíos en la articulación de sus componentes (ver figura 1). A nivel de sistema educativo se espera que el programa: aumente el salario docente según desempeño individual, retenga a buenos docentes en el aula e incentive la formación de pares, en tanto en el largo plazo se espera que AEP contribuya al mejoramiento de la calidad educativa. A nivel establecimiento se espera que los profesores certificados estimulen a sus pares a acreditarse y que modelen buenas prácticas entre sus colegas.

En el mediano plazo, se espera que los docentes acreditados contribuyan a instalar el concepto de calidad en los establecimientos. Por último, a nivel individual se espera que los acreditados aumenten su salario, perciban el reconocimiento social e incrementen su autoestima profesional, mientras que en a largo plazo se espera que la Acreditación estimule la reflexión de los docentes sobre sus propias prácticas educativas (Araya, Taut, Santelices, Manzi \& Miño, en prensa).

Figura I

Teoría de Acción de AEP

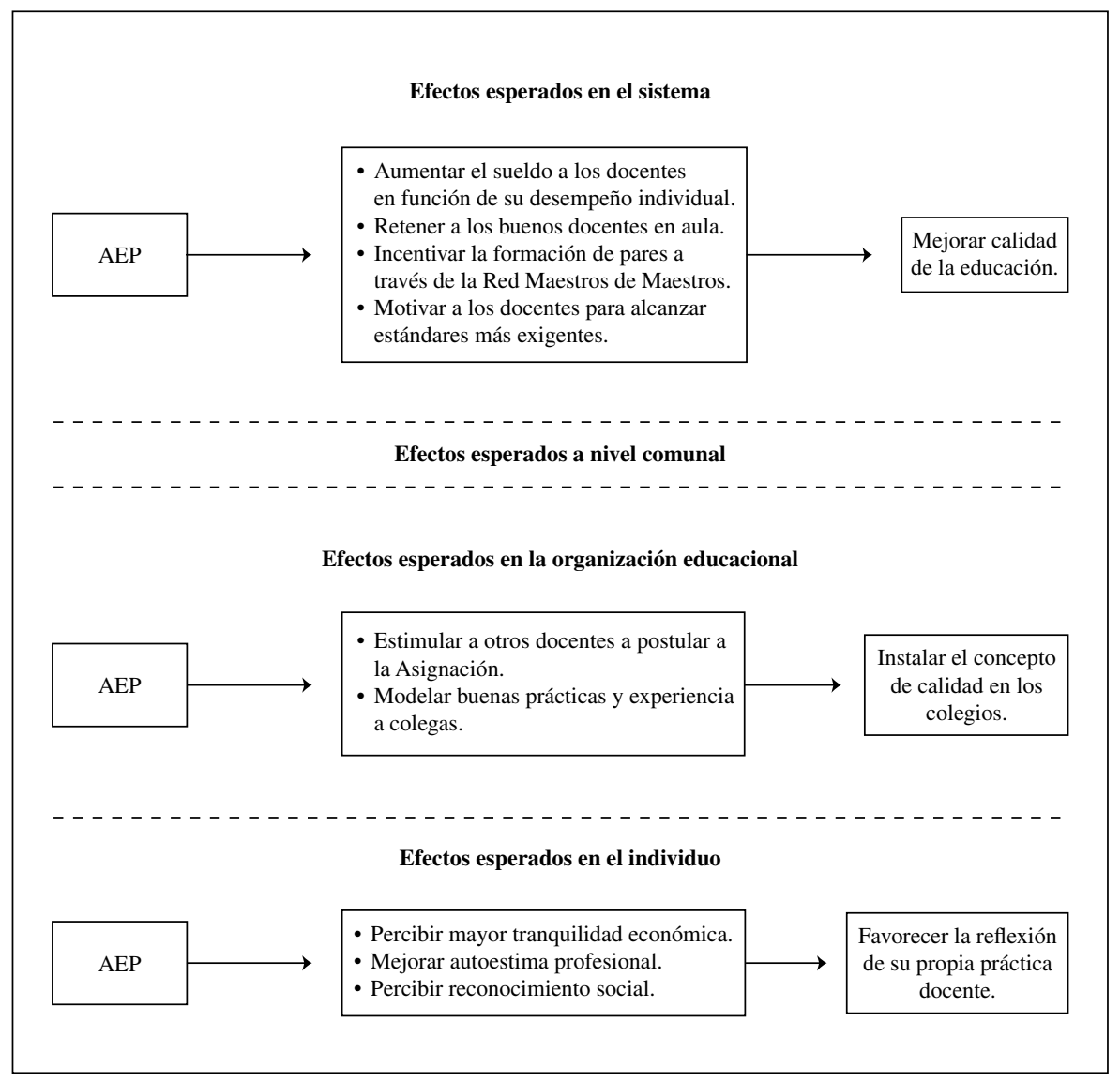


El estudio que se presenta a continuación, tiene como propósito verificar empíricamente la teoría de acción del programa para comprobar si se están produciendo los efectos esperados por los creadores de AEP, junto con identificar efectos -positivos o negativosque no hubieran sido anticipados originalmente. Dicho de otro modo, el estudio busca aportar antecedentes para juzgar la validez consecuencial del Programa AEP.

\section{MÉTODO}

El estudio se realizó a través de metodologías cualitativas, cuyos procedimientos y técnicas permiten abordar empíricamente interrogantes sobre percepciones subjetivas de los participantes del estudio. En ese sentido, estas metodologías ofrecen estrategias de generación de conocimientos que privilegian la perspectiva de los docentes que postulan a la acreditación y de los directivos de los establecimientos que ejercen roles de supervisión sobre estos docentes. En otras palabras, interesa describir los efectos que ha tenido AEP sobre los docentes y las escuelas desde el marco de referencia y contexto en que se ubican los propios actores.

\subsection{PARTICIPANTES}

Para el estudio se utilizó un muestreo intencionado a partir de ciertos criterios que establecen presunciones sobre la tipicidad y distribución de los postulantes a la Asignación (Flick, 2004; Patton, 2002). Uno de los criterios empleados para intencionar la muestra tuvo que ver con la accesibilidad a los participantes. De esta forma, solo se incluyeron docentes y directivos que se desempeñaban en establecimientos de la región metropolitana con dependencia municipal y particular subvencionada.

Adicionalmente, se intentó conservar cierta representatividad de la distribución de los postulantes a AEP según la dependencia administrativa de los establecimientos en que trabajan.

El análisis de las bases de datos de los candidatos de AEP arroja que -en promedio- $2 / 3$ de los postulantes se desempeñan en establecimientos municipales y el resto en establecimientos particulares subvencionados (ver aep. mineduc. cl).

En consecuencia y siguiendo una estructura anidada, se seleccionaron diez comunas del Gran Santiago. Al interior de cada comuna, se seleccionaron tres establecimientos: dos municipales y un particular subvencionado. De esta forma, la muestra quedó constituida por treinta establecimientos, siendo veinte de ellos escuelas o liceos de administración municipal y diez colegios particulares subvencionados.

En cada establecimiento, fueron entrevistados el (la) director(a) y el(la) Jefe de UTP. Por consiguiente, se efectuaron 60 entrevistas a los directivos de los establecimientos seleccionados. Asimismo, se entrevistaron 10 docentes de esos establecimientos que postularon y no obtuvieron la Asignación y 35 docentes que sí la obtuvieron. Estos últimos fueron entrevistados en la modalidad de grupos focales.

\subsection{PROCEDIMIENTOS}

Con los directivos de cada establecimiento se realizaron entrevistas individuales y semi-estructuradas entre noviembre del 2008 y abril del 2009. Las entrevistas tuvieron una duración aproximada de una hora y fueron grabadas en audio. 
Con los profesores acreditados, por su parte, se realizaron cinco grupos focales a fines del 2009. Los grupos fueron homogéneos en cuanto a tipo de dependencia administrativa y NSE de la comuna en que se ubican los establecimientos en los que se desempeñaban los profesores convocados. Cada grupo focal fue realizado por una dupla de investigadoras, estando una a cargo del registro y las notas de campo, mientras la otra asumía el rol de moderadora de la discusión. Los grupos focales duraron aproximadamente dos horas y fueron grabados en audio.

Con los postulantes que no obtuvieron la acreditación, se realizaron entrevistas individuales semi-estructuradas en agosto del 2010. Cada entrevista duró aproximadamente una hora y fue registrada en audio.

Todas las grabaciones en audio fueron transcritas a texto, procurando conservar con la máxima integridad los distintos elementos del discurso oral de los participantes.

Los datos reunidos fueron analizados siguiendo un análisis cualitativo de contenido (Hsieh \& Shannon, 2005). Para ello, se analizaron preliminarmente algunas entrevistas y grupos focales transcritos a fin de construir un esquema de códigos, que luego fue aplicado en forma iterativa a la totalidad del material transcrito.

Cada entrevista y grupo focal fue codificado por un par de investigadoras con el propósito de triangular los resultados de la codificación y cautelar los criterios de credibilidad y auditabilidad propuestos por Guba \& Lincoln (Guba \& Lincoln, 1981 en Castillo \& Vásquez, 2003).

\section{RESULTADOS}

En este apartado se describen las consecuencias del Programa AEP de acuerdo a la percepción de los directivos y docentes postulantes que participaron en el estudio.

Para efectos de la presentación, los hallazgos se agrupan en tres categorías. En la primera, se describen los efectos del programa en el docente que postula a la certificación, tanto si la obtiene como si fracasa en su intento, mientras que en la segunda categoría se exponen los efectos del programa al interior de la organización escolar. Finalmente, en la tercera categoría se presentan algunos hallazgos preliminares sobre el impacto de la certificación a nivel del sistema educacional.

Al interior de cada categoría, los resultados se clasifican en efectos que coinciden con la Teoría de Acción del programa o en efectos emergentes. En el primer caso se trata de efectos esperados o intencionados por los creadores de AEP. En el segundo, se describen aquellas consecuencias que no fueron intencionadas o previstas por las personas que participaron en el diseño y puesta en marcha del programa.

\subsection{EFECTOS A NIVEL INDIVIDUAL}

\subsubsection{Efectos esperados en los docentes acreditados según Teoría de Acción de AEP}

Una de las consecuencias observadas a nivel individual que reciben sólida evidencia empírica refiere al fortalecimiento de la autoestima profesional de los profesores certificados. La totalidad de los profesores entrevistados estiman que haber obtenido la Asignación 
ha mejorado la valoración profesional de sí mismos y su sentido de autoeficacia docente. Declaran sentir mayor confianza en sus capacidades, sentirse responsable de hacerlo bien y tener el deseo de apoyar a los pares. Además, experimentan mayores niveles de autoexigencia, deseos de perfeccionarse, mayor interés por innovar en prácticas de enseñanza y adecuarse a necesidades de aprendizaje de sus estudiantes, como resultado de obtener la certificación.

Los docentes declaran sentirse orgullosos de sí mismos por haber obtenido una certificación que es reconocida en el sistema escolar como un proceso exigente y arduo: "es un esfuerzo personal, también el anhelo de querer mejorar, cambiar. Por eso para mí esto ha significado un cambio positivo" (F2, 418). "Ellos sabían que esto era una cosa difícil, o sea, que estaba hecha así porque era un proceso selectivo, no todos iban a llegar hasta final" (F2, 218).

Un tercio de los directivos y la mayoría de los docentes entrevistados perciben que la certificación no ha implicado una mejoría en el desempeño del profesor: simplemente confirma un desempeño que ya era bueno antes de la certificación:

"Yo me valoro mucho, siempre me he encontrado buena profe. Pero después de la AEP, fue como reafirmar(lo)" (F3, 488).

"en el fondo, tener el certificado le demuestra a los otros (que eres un buen profesor). Porque yo creo que todos, cuando postulamos, (lo hacemos) confiando en nuestro trabajo" (F3, 491).

Con todo, un tercio de los directivos y algunos docentes acreditados registran cambios en su nivel de profesionalización, en la medida que emplean un lenguaje más técnico, muestran mayor sistematización de sus planificaciones de clase y una mayor reflexión sobre su propia práctica pedagógica:

"Es lo mismo que tú hacías (antes de la acreditación,) pero que está (más) estructurado, está ordenadito. Esto para mí ha sido (optimizar una práctica), así lo considero. Porque todos planificamos, todos ejecutamos clases, pasamos materia, inventamos leseras, buscamos cosas, pero de repente somos como medios dispersos. En cambio ahí te obligan: ordénate, ordénate, ordénate... iy sirve!” (F1, 73).

" $Y$ uno aprende, a través de este proceso, que es súper importante la autoevaluación. Estar dándose cuenta de las cosas malas que estás haciendo, de tus malas prácticas, para poder transformarlas" (F2, 343).

"lo veo como una instancia de aprendizaje (...) porque demanda tiempo, demanda pensar en temas de educación. No es posible trabajar en un portafolio y (que no influya) en lo que uno está enseñando (...) yo no lo disocio” $(F 2,25)$.

En relación a que el incentivo económico que reciben los profesores acreditados les otorgaría cierta tranquilidad económica, un cuarto de los directivos entrevistados declara estar de acuerdo. Los acreditados, en cambio, relatan tantas dificultades para su obtención, que finalmente pierde su valor como premio. La totalidad de los profesores acreditados que participaron en el estudio reconoce permanentes atrasos en el pago del bono, atribuyéndolo a la burocracia que deben sortear y a la desinformación en los distintos niveles del sistema administrativo respecto a cómo opera el pago: 
“Da vergüenza... porque hay que llamar a la provincial y es como (jugar) al 'compra huevos'... Tú llamas al CPEIP (y te dicen) nosotros enviamos la lista al SEREMI. El SEREMI (dice), no, nosotros lo mandamos a la provincial. La provincial (dice) no, usted no puede retirar el dinero porque tiene que retirarlo su empleador. $Y$ el empleador dice vamos a retirar todos de una sola vez" (F5, 1061-1063).

"Te dicen (que recibirás el pago) en junio y en diciembre [risas suaves]. Y tú sabes que es un decreto de ley, que se supone la ley se tiene que cumplir [enfatiza]. Y pasa junio, pasa julio y empezar a llamar al CPEIP... es humillante, porque después ya te tratan pésimo en el CPEIP [risas]" (F3, 135).

"es desagradable ¿sabes?, porque esto es algo tuyo. Es humillante, porque es algo TUYO [enfatiza]. Y, por otro lado, le llega al sostenedor y el sostenedor te lo traspasa a ti ¿Por qué?, si es algo que tú hiciste, (por qué) no te llega directamente”. (F3, 137).

Mención aparte requiere la expectativa de que AEP concediera reconocimiento social a los profesores destacados por la calidad de su trabajo. Desde la percepción de los docentes acreditados, la reacción de la comunidad escolar es ambivalente: al conocerse los resultados del proceso de certificación los pares y directivos hacen públicas sus felicitaciones a los postulantes exitosos, sin embargo también aparecen indicios de envidia y desconocimiento de los logros obtenidos.

Aún cuando señalan sentirse respetados por colegas que pertenecen a su red más cercana, casi la totalidad de los profesores que participaron en el estudio reportan haberse sentido aislados o criticados por sus pares, algunos de los cuales siembran dudas sobre la legitimidad de la certificación o de los reales méritos de profesor acreditado. Los directivos de un tercio de los establecimientos concuerdan con esta percepción.

"Al final me fui de ese colegio por lo mismo, porque esto genera malestar en los profes, porque postulaban los otros y no quedaban. Entonces, había un momento en que estaba casi sola, como que te aíslan y siempre te andan mirando" $(F 2,102)$.

De un modo paradójico, algunos profesores acreditados declaran sortear las dificultades relacionales que les ha acarreado su certificación volviéndose "invisibles" y escondiendo su condición de acreditados. Así, esperan prevenir el rechazo y aislamiento de sus colegas: "A nosotros, esta cosa de la acreditación nos trajo problemas. (...) Cuando nos acreditaron nos regalaron una chapita, una cosita que te acredita como profesor de excelencia... No nos podíamos colocar nuestra identificación. Generaba envidia, molestia, quizás" (F2, 48-55).

Respecto a las autoridades escolares, la mayoría de los acreditados dice haber recibido algún tipo de reconocimiento público por parte de los directivos del establecimiento al momento de comunicar el logro de la certificación. Pocos mencionan haber recibido algún reconocimiento de los sostenedores, y son aún menos los que consideran que el reconocimiento inicial se transformó en nuevas oportunidades y desafíos laborales al interior de la escuela o del municipio.

En síntesis, los docentes acreditados señalan haber recibido un reconocimiento inicial de su comunidad escolar local (escuela y sostenedores), pero se trataría de un reconocimiento superficial, ambiguo y no perdurable en el tiempo. 


\subsubsection{Efectos emergentes en los docentes acreditados}

Entre los efectos individuales no intencionados pero que son percibidos como positivos por los entrevistados se menciona cómo AEP amplía las oportunidades de carrera profesional de los docentes. Un quinto de los directivos y de los docentes acreditados refieren que la certificación ha favorecido la movilidad laboral al interior de la escuela, implicando una extensión de la jornada laboral de los docentes. No obstante, la mayoría de los profesores acreditados señalan que la certificación ha mejorado sus oportunidades de movilidad laboral fuera de la escuela, en la medida que les permite establecer contacto con organizaciones y redes del ámbito educacional más amplio. Asimismo, dos tercios de los acreditados que participaron en el estudio declaran que la certificación les ha dado acceso a más oportunidades de perfeccionamiento (pasantías y mejores condiciones para postular a becas):

"Se te abren otras puertas, de postular a cosas, de optar, de interactuar con otras personas, por ejemplo..." (F3, 131).

"(Ahora, perfeccionamiento al que postulo, perfeccionamiento que me lo dan. En ese sentido, yo creo que (tener $A E P$ ) me ha ayudado bastante. $O$ sea, yo postulo a diplomados, me lo financian completamente, magíster también postulo y ahí está la posibilidad (de conseguir financiamiento $) "(F 3,305)$.

En un par de casos, la acreditación ha funcionado como trampolín para los profesores, quienes renuncian a la certificación para trabajar en colegios particulares pagados con mejores condiciones de salario y/o para enseñar a estudiantes menos vulnerables desde un punto de vista socioeconómico. Nuevamente, esta consecuencia resultaría paradojal por cuanto los buenos profesores acaban enseñando a estudiantes más aventajados en su capital cultural, en lugar de atender a estudiantes que requieren con mayor urgencia de sus competencias pedagógicas de excelencia.

Otro efecto no intencionado que resulta interesante dice relación con la fuente de reconocimiento social para los acreditados. Tal como se señaló previamente, la evidencia reunida refleja que los acreditados no se perciben claramente reconocidos por sus pares y jefes. Reportan, en cambio, sentirse genuinamente valorados por otras instancias del sistema educacional, externas su comunidad local y a las cuales no habían tenido acceso antes de la certificación. Mencionan haber sido convocados por universidades, por el Ministerio de Educación o por editoriales que los llaman a participar como autores o evaluadores de textos escolares:

"Yo sentí que se me reconoció mucho más fuera del colegio, que en el colegio o la comuna" $(F 1,106)$.

"De repente te llaman para participar en los ajustes curriculares y te pagan. Entonces, ellos quieren profesores $A E P ”(F 3,142)$.

En términos de su práctica profesional, la mayoría de los docentes acreditados experimentan gran responsabilidad por mantener estándares elevados de desempeño y cumplir con las expectativas de su entorno. Esto se ha traducido en altos montos de auto-exigencia:

"Yo siento que es algo personal, es algo que no sé si a todos les ha pasado, pero me siento con la responsabilidad de mantener un buen nivel” (F5, 836). 
“ ¿Cómo tomo el ser de AEP? Lo tomé como una responsabilidad. Entonces eso me ha hecho seguir tomando cursos, especializarme en matemáticas, que era el ramo que yo tenía, me especialicé en eso, y bueno, cuando hice la pasantía, la pedí en matemáticas también... Me he ganado el respeto de los niños" (F1, 282).

"te vas dando cuenta que te exiges más [enfatiza], o sea ya, si no sé esto, me trato de perfeccionar, voy a tratar de saber más, porque si alguien te viene a preguntar, sobre todo las chiquillas que están recién empezando, ¿cómo no las vas a orientar bien?” (F3, 129).

"el hecho de tener la AEP, significa que uno tiene que estar como más actualizada, porque siempre te andan preguntando" $(F 3,242)$.

Ocasionalmente, estos elevados niveles de auto-exigencia les generan conflictos internos; pues se sienten sobrecargados y permanentemente observados por sus pares.

\subsubsection{Efectos emergentes en candidatos que no obtuvieron la certificación}

Cabe mencionar que desde los creadores de AEP no se esperaba producir efectos en los candidatos a la certificación por el mero hecho de postular a ella. Por eso, todos los hallazgos que se exponen en este apartado son emergentes.

En el curso de la investigación con los profesores acreditados aparecen antecedentes que indican una valoración importante del proceso de postulación. En opinión de estos docentes, la postulación permite adquirir herramientas útiles para la práctica profesional, más allá del logro de la certificación. Surge la interrogante de si esta percepción es válida también para los candidatos que no obtuvieron la Asignación.

Las entrevistas con los postulantes que no obtuvieron la certificación confirman la valoración que los profesores hacen por haber participado en esta evaluación. El hecho de transitar por el proceso de elaborar un portafolio que documente evidencias de su quehacer pedagógico y rendir una prueba de conocimientos específicos sobre la disciplina que se enseña, impacta positivamente en sus prácticas laborales.

En particular, siete de los diez entrevistados señalan que realizar el portafolio favoreció la reflexión sobre las propias prácticas, ayudándolos a identificar las propias falencias. En segundo lugar, consideran que realizar el portafolio les permitió adquirir herramientas de planificación que han redundado en optimizar el tiempo lectivo y estructurar mejor las actividades de aprendizaje.

La mitad de los entrevistados señala que la postulación los obligó a estudiar y revisar con detalle los documentos oficiales del Ministerio y literatura sobre reforma educacional. Para un tercio de los entrevistados, la postulación y su consecuente fracaso los motivó a buscar perfeccionamiento para aquellos aspectos deficitarios de su práctica.

Al preguntarles si intentarían nuevamente conseguir la certificación, 8 de los profesores contestaron afirmativamente.

Al recibir los malos resultados, la mitad de los entrevistados manifiesta haber experimentado frustración y desencanto pasajero, particularmente por el gran esfuerzo y sobrecarga de trabajo que les significó cumplir con los requerimientos de la Asignación.

No obstante, la mayoría atribuye el fracaso a las propias falencias en la elaboración del portafolio. 


\subsection{EFECTOS A NIVEL ESCUELA}

\subsubsection{Efectos esperados en la organización escolar según Teoría de Acción de AEP}

El efecto esperado a nivel organizacional que recibe mayor soporte empírico refiere a la motivación que genera el docente acreditado en sus pares para postular a la certificación en el futuro. Los directivos de un tercio de los establecimientos y los docentes acreditados de la mayoría de los grupos focales confirman esta impresión.

Sin embargo, los directivos señalan que la magnitud de motivación disminuye cuando en el mismo establecimiento hay postulantes que no obtuvieron la certificación.

En cuanto a la intención de que el programa sirviera para diseminar las buenas prácticas y experiencias pedagógicas de los profesores acreditados con sus colegas, la evidencia resulta poco contundente. Cuando hay experiencias de intercambio de prácticas profesionales, por lo general, responden a iniciativas de los mismos docentes. No son espacios institucionalizados en la escuela: "a mí se me ha pedido ayuda: ¿tú puedes ayudarnos con esto? (...) y yo estoy siempre presta a ayudar, puedo estar muy cansada, pero digo sí. Pero es como muy voluntario el trabajo. Y una que otra vez me han pedido esa instancia de trabajar con pares" (F2, 263).

En los establecimientos de dependencia municipal, los profesores acreditados son especialmente requeridos por sus pares para apoyar la preparación del portafolio exigido por el Sistema de Evaluación del Desempeño Docente (SEDD): "por ejemplo ahora que ellos están haciendo la evaluación docente, me preguntan y qué opinas de esto y qué crees de esto otro" (F1, 192); "algunas amigas me han pedido que les revise el portafolio" (F3, 81).

Se puede hipotetizar que la falta de espacios colaborativos de reflexión y planificación de clases entre los docentes acreditados y sus pares se debe, en parte, a que AEP es visto por los directivos como un incentivo exclusivamente individual. En consecuencia, el grueso de los directivos entrevistados no utiliza la información generada por el programa como insumo para la gestión pedagógica. Por otro lado, tampoco se sienten comprometidos a incentivar la postulación de sus docentes a la certificación, restringiendo su rol a la transmisión de información recibida desde el nivel central.

Solo en un tercio de los establecimientos incluidos en el estudio, los directivos mencionan asignar responsabilidades críticas a los profesores acreditados que les permitan compartir sus prácticas con otros. Entre las nuevas responsabilidades asignadas a los acreditados se describen responsabilidades técnicas $\mathrm{u}$ otras ocupaciones que resulten compatibles con el trabajo de aula, a saber: jefaturas de departamentos, jefaturas de curso, "preparación" del Simce, supervisión y observación de clase, apoyo en la formulación de proyectos institucionales. Algunos profesores acreditados lo confirman:

"Yo acompaño, también, al grupo de matemática del colegio, también trabajo con ellos en el departamento de matemática, acompaño al UTP" $(F 3,242)$.

"Yo siento que en el colegio, he sido valorada por el trabajo que realicé, se me ha dado la oportunidad de trabajar con los profesores. El año 2005, el colegio me ofreció la oportunidad de formar parte del equipo de UTP. En ese sentido yo siento que ha sido súper bien valorado mi trabajo, y siempre estoy ahí, cualquier cosa pedagógica, estoy ahí presente" $(F 3,75)$. 
Los acreditados que trabajan en establecimientos particulares subvencionados agregan que se les ha encomendado ser mentores de estudiantes de pedagogía en práctica y que se les pide opinión cuando el establecimiento quiere presentar un proyecto de mejoramiento educativo al Ministerio de Educación. "la directora me dijo ¿y por qué la profesora de religión? Porque ella trabajó en el portafolio (le dije) ¿Pero ella sabrá? Si es capaz de hacer un portafolio es porque tiene el vocabulario técnico suficiente como para poder trabajarlo (contesté). Y se lo pedimos. Ella estaba muy contenta y muy agradecida que la hayamos considerado. De hecho, nos ganamos el proyecto de mejoramiento educativo, que fueron varios millones para el colegio" (F2, 271).

Solo en uno de los grupos focales -de acreditados que trabajan en colegios particulares subvencionados- los docentes mencionan que los directivos les permitían trabajar con mayor autonomía luego de obtener la certificación.

\subsubsection{Efectos emergentes en la organización escolar}

Como un efecto inesperado y negativamente valorado por los profesores acreditados se menciona la indiferencia de los directivos a la certificación. En efecto, dos tercios de los acreditados que participaron en el estudio plantean que desde las instancias directivas no se generan oportunidades para que los acreditados desplieguen su potencial liderazgo pedagógico:

"Yo creo que ahí uno ve el reflejo de liderazgos que están mal apreciados, mal percibidos por parte de las direcciones de los colegios. Porque en el fondo, nosotros, las personas acreditadas hemos demostrado esas capacidades de liderazgo. Entonces, las personas que tiene ciertas capacidades debieran asumir ciertas responsabilidades y tus jefes deberían estimularte para que siguieras promoviendo eso, el trabajo en equipo, etc. Y no es así. $O$ sea, te van bajando el perfil, es mejor irlos acallando, porque muchos de ellos, o nosotros, somos motivos de conflicto, porque (si haces una) crítica dentro del colegio, la crítica siempre es percibida como algo negativo, como algo que va contra del sistema" (F2, 341).

En relación a esto último, algunos señalan haber sostenido abiertos conflictos con las autoridades de sus establecimientos. Si bien esta experiencia es reportada con escasa frecuencia, un par de docentes señalan haber sido hostilizados por los jefes de UTP de sus establecimientos, quienes sentirían amenazada su autoridad técnica por el actual status de los docentes cuyas competencias fueron certificadas: "Finalmente, a mí me designaron con horas de la Unidad Técnica, yo estoy con catorce horas en UTP (...) pero igual se generó como una amenaza -yo lo visualicé así- como en las tinieblas, para la Jefa Técnica -¿Qué tú le aserruchabas el piso?- ¡Claro!, exactamente” (F5, 597-599).

"Ahora se produjo un problema mayor porque al traspasarme como coordinadora de bachillerato internacional surgió la idea de ir a capacitarme a México. Entonces, la directora de educación me mandó a México (...) Y eso generó aún más problema (en el Liceo) de por qué no fue la Jefa Técnica y por qué fui yo” (F5, 613-617).

Desde la otra cara de la moneda, estos hechos son relatados por los directivos como un efecto negativo de la certificación, según el cual algunos profesores acreditados adoptarían una actitud soberbia e inflexible con sus pares y jefes. 


\subsection{EFECTOS A NIVEL SISTEMA EDUCACIONAL}

Aún cuando la magnitud del estudio no permite ser concluyentes sobre el impacto de AEP a nivel del sistema educacional, aporta luces que deben ser investigadas a mayor escala para determinar si constituyen tendencias.

Los creadores del programa esperaban que a nivel sistema AEP contribuyera a aumentar el sueldo de los docentes en función de su desempeño individual. Adicionalmente, esperaban que el programa ayudara a retener a los buenos docentes en el aula, incentivara la formación entre pares a través del acceso de los acreditados a la Red de Maestros de Maestros (RMM) y motivara a los docentes para alcanzar estándares más exigentes.

Los datos recolectados en este estudio permiten apoyar preliminarmente estas expectativas.

En relación a la retención de buenos docentes en el aula, la información obtenida a partir del análisis de la trayectoria profesional post-acreditación de los docentes participantes, indica que la mayoría se mantiene en aula pero realiza -simultáneamente- otras funciones dentro o fuera de la escuela. De esta forma, mantienen los beneficios económicos de la certificación y al mismo tiempo realizan asesorías y apoyo técnico en sus establecimientos o en otras escuelas y liceos. Son convocados también para tareas específicas por el Ministerio de Educación, tales como validar preguntas del SIMCE o ser evaluador par en el SEDD.

Con todo, 7 de los 35 profesores entrevistados cambia de trabajo después de certificarse. Cinco de ellos han dejado definitivamente el trabajo de aula, aunque siguen ligados a la educación, desempeñándose en instituciones terciarias, en programas del Ministerio de Educación o emigrando a cargos directivos en el área técnico-pedagógica de sus establecimientos. Los otros dos profesores siguen en aula pero declaran haberse cambiado a colegios de dependencia particular subvencionada en búsqueda de mejores condiciones laborales:

"En el momento (de recibir AEP) quizás no me generé muchas expectativas. Con el tiempo me doy cuenta que mi colegio no tenía expectativas. Sin embargo, exteriormente, los que te están observando, ellos sí generan expectativas. Y se me abrieron muchas puertas, de hecho consolidé un trabajo en el CIDE y seguí trabajando en investigación ahí, asesorando didácticamente en muchos colegios y después me invitaron a trabajar en la formación de profesores en la Universidad XX, en los programas de pedagogía para profesionales”. (F5, 637-638).

Respecto a la RMM a la que los acreditados pueden postular previa realización de un portafolio, hay que señalar que pocos lo intentan. Hasta el año 2009, de los 3477 profesores acreditados solo el 30\% había ingresado a la Red (Fuente: aep. mineduc. cl y rmm. cl). Si bien la mayoría de los entrevistados la considera una iniciativa valiosa que les permite compartir sus saberes y competencias con otros profesores, estiman que les demanda mucho tiempo fuera de su jornada laboral. Algunos de los profesores entrevistados que pertenecen a la Red indican que la remuneración asociada a la participación en proyectos institucionales es insuficiente; en tanto la contribución con material didáctico en el portal virtual de la red no tiene remuneración alguna y apela a la buena voluntad del docente:

"Yo también estoy, pero no estoy actualizada. Yo tengo que reconocer que me he dedicado más a lo del colegio y lo de la red... empecé muy embalada (sic), metiendo mucha información 
a la red. Y después la tengo ahí, como se dice, no actualizada. Por una razón (de falta) de tiempo solamente, me he dedicado más al colegio”. (F3, 640).

"Me he dado cuenta de un par de cosas que son bien significativas. Yo soy bastante crítico pero mi percepción es lo siguiente, creo que (con) este título honorífico, (...) te habilitan una página web para que tú prepares a los profesores a nivel nacional y publiques guías y qué sé yo, ese tipo de cosas. Entonces tú tienes que hacer un trabajo completamente voluntario, en tu casa ¿no? Para colaborar generosamente”. (F5, 1148).

Una docente explicita una opinión que circula entre los entrevistados respecto a elementos de la cultura docente que hace difícil implementar la formación entre pares: "yo he sido de repente bastante crítica de nuestro quehacer, porque lo he vivido de alguna manera. Los profesores somos muy reacios a que un profesor nos dé un curso: ¡No! ¿Un par nos entrega un curso?, se escuchan muchas críticas y más o menos ¿qué se creen? ¿A quién le ganó? No es cien por ciento, no es siempre, pero a veces ocurre”. (F1, 305).

\subsection{VARIABLES QUE EXPLICAN DIFERENCIAS EN LOS EFECTOS PERCIBIDOS DE AEP}

Con los datos aportados por los directivos y profesores entrevistados, se clasificaron los 30 establecimientos en tres categorías que buscan describir la magnitud de los efectos del programa sobre la comunidad escolar. De esta forma, se distinguió entre escuelas donde el programa parece tener un efecto promisorio, un efecto básico y donde no tiene ningún efecto. En el caso del "efecto promisorio", los profesores se sienten reconocidos por su comunidad y los directivos usan la información generada por la certificación para asignar tareas críticas a los profesores acreditados. Nueve escuelas califican en esta categoría.

Trece establecimientos califican como "de efectos básicos", vale decir, escuelas y liceos en los que se aprecian algunas señales de reconocimiento inicial a los docentes acreditados pero que no implican ningún cambio en sus funciones o bien, se aprecia ambivalencia hacia el docente acreditado. En estos establecimientos, los directivos restringen su rol a difundir entre los docentes la información sobre la postulación al programa.

En los ocho establecimientos que caen en la categoría "sin efecto", los directivos declaran no percibir el programa como parte de su gestión y la reacción de los pares es indiferente y a veces hostil.

Al agrupar los establecimientos en estas categorías, las escuelas con un efecto promisorio parecen compartir -en mayor medida- un clima organizacional percibido como nutritivo; mientras que en aquellos establecimientos en que el programa no funciona o no se perciben efectos favorables, predomina la percepción de climas sociales deteriorados. Dicho de otra forma, la variable "clima social escolar" impresiona como una variable que mediatiza los efectos de AEP en el establecimiento.

Se entiende por clima social la percepción que tienen los individuos de los distintos aspectos del ambiente donde desarrollan sus actividades habituales (Arón y Milicic, 2000). De acuerdo a estas autoras, los climas sociales escolares nutritivos son aquellos que generan entornos "en que la convivencia social es más positiva, en que las personas sienten que es agradable participar, en que hay una buena disposición a aprender y a cooperar" (Op. Cit, p. 118). Los climas sociales tóxicos, en cambio, serían aquellos que contaminan un ambiente impregnado por los aspectos más negativos. Se invisibilizan 
los aspectos positivos, los que aparecen como inexistentes y se instala una percepción sesgada que amplifica la apreciación de los aspectos negativos (Ibid).

\section{CONCLUSIONES}

Tomado la teoría de acción de AEP como referente para contrastar los efectos observados de AEP, se puede resumir que algunas expectativas a nivel individual son las que cuentan con mayor soporte empírico. Los profesores acreditados dicen experimentar mayor autoestima profesional y declaran que el incentivo económico es bienvenido, aunque los obstáculos burocráticos para recibirlo le restan valor.

En cuanto al reconocimiento social percibido, los hallazgos revelan que los docentes se sienten más reconocidos por instancias externas y distantes de su entorno local que por su propia comunidad escolar. El reconocimiento de los sostenedores, directivos y pares resulta más bien exiguo y ambivalente. Aparentemente, la distinción de algunos docentes entre el colectivo aumenta la distancia social entre el profesor destacado y sus pares. Este hallazgo se asemeja a lo encontrado por el National Research Council (2008) en EE.UU. De acuerdo a esta investigación, profesores certificados por el NBPTS hacen considerables esfuerzos para minimizar las distinciones entre ellos y sus colegas no certificados, a veces incluso llegan a ocultar el hecho de que se han ganado la credencial. De alguna forma, los profesores certificados evitan transgredir la tradición igualitaria que domina el ámbito docente a fin de evitar el aislamiento en sus escuelas (Koppich et al., 2006; Sikes et al., 2006; Yankelovich Partners, 2001 en National Research Council, 2008).

La falta de reconocimientos de la comunidad local influiría en que a nivel de establecimiento la expectativa de diseminar las buenas prácticas de los acreditados entre sus pares no se cumpla. Además, incide el que los directivos mayoritariamente se sientan descomprometidos con el programa y lo valoren solo como un incentivo con impacto individual. El recurso docente certificado es pobremente utilizado en la gestión pedagógica que realizan los directores y sostenedores.

Hay evidencias de que la presencia de acreditados en el establecimiento estimula a otros a postular. Probablemente el éxito de algunos ayude a validar la asignación como un incentivo alcanzable si se hacen los esfuerzos necesarios.

Respecto a los efectos esperados a nivel sistema, los datos deben ser considerados con cautela por dos razones: se requieren nuevas investigaciones con muestras de mayor magnitud para establecer tendencias y segundo, porque los efectos a nivel sistema se constatan en periodos más largos de tiempo. Con todo, la evidencia disponible revela que los profesores acreditados parecen mantenerse mayoritariamente en el aula. Unos pocos cambian de rol pero se mantienen ligados al sistema educacional de alguna manera. Este resultado es consistente con lo reportado por Sykes et al. (2006) con los profesores certificados por el NBPTS en EE.UU.: al comparar acreditados con no acreditados encontró que es más probable que los primeros planeen permanecer en la docencia en lugar de abandonar la enseñanza (Sykes et al., 2006 en National Research Council, 2008).

Respecto a favorecer la formación entre pares, haciendo accesible la participación de los acreditados a la Red Maestro de Maestros, no parece haber prueba suficiente de que los docentes se estimulen a participar en la Red y por tanto ejerzan formalmente el rol de mentor. 
Los resultados de esta investigación permiten sugerir la necesidad de introducir algunos cambios en el diseño e implementación del programa AEP a fin de optimizar su efecto sobre el sistema educacional y los actores que lo constituyen. Por lo pronto, es difícil que lo acreditados tengan un efecto significativo a nivel de establecimiento y que estos reverberen a nivel sistema si no se involucra de un modo más activo a los sostenedores y a los directivos de las escuelas y liceos. Al fin de cuentas, son ellos quienes crean las oportunidades para que los acreditados puedan desplegar y ejercer un liderazgo técnico entre sus pares. Esto último es coherente con los hallazgos de Fullan (2004) al revisar cambios educativos a gran escala que han resultado exitosos. En opinión de este autor los esfuerzos de cambio deben ser implementados tanto en el docente como en sus contextos, articulando esfuerzos consistentes y alineados en todos los niveles del sistema. Desde este punto de vista, el supuesto de la teoría de acción de AEP, según el cual los docentes acreditados formarían una suerte de masa crítica al interior de sus establecimientos, promoviendo cambios en las prácticas pedagógicas que se propaguen a nivel de sistema, resulta ingenuo e improbable. Individuo y sistema deben cambiar a la par.

Para favorecer los efectos esperados de AEP a nivel sistema y a nivel escuela, se requiere el compromiso firme de las autoridades educativas municipales y del establecimiento no solo para incentivar la postulación de sus profesores a la certificación, sino también para generar las condiciones necesarias que permitan aprovechar las capacidades de los acreditados e instalar comunidades de aprendizaje entre los docentes. Esto supone un cambio de perspectiva respecto a los enfoques de liderazgo prevalentes, en la medida que define el liderazgo efectivo como aquel orientado a fortalecer la capacidad de decisión de otros actores de la escuela. En ese sentido, un liderazgo efectivo es aquel que se distribuye a través de la organización (Fullan, 2004).

Los docentes acreditados pueden ser un recurso fundamental para la creación de capacidades al interior de sus escuelas y en la comunidad local. Sus competencias pedagógicas pueden ser aprovechadas para establecer comunidades docentes en que sus miembros aprenden unos de otros. De ese modo, se establecerían oportunidades de desarrollo profesional docente similares a aquellas que han demostrado mayor eficacia, vale decir, las que implican actividades de aprendizaje activas y en el mismo lugar de trabajo, como grupos de estudio, proyectos de investigación-acción y portafolios, entre otras. Las oportunidades de desarrollo profesional deben transcurrir en un "proceso de colaboración", que es más eficaz cuando hay interacciones significativas entre los profesores (Villegas-Reimers, 2003). Por otra parte, los estudios indican que cuanto más tiempo se le da a los profesores para la planificación, debates y otras actividades de desarrollo profesional, los docentes son más eficaces para enseñar $\mathrm{y}$, por consiguiente, los estudiantes aprenden más (Darling-Hammond, 1999).

Los hallazgos aquí reportados proporcionan evidencia inicial pero relevante para evaluar la validez consecuencial del programa. Quedan preguntas importantes por responder, especialmente para verificar efectos de AEP a nivel de sistema educativo. Estas preguntas requieren otro tipo de diseño metodológico. En el futuro será importante, por ejemplo, valorar el impacto de AEP sobre las prácticas pedagógicas de los docentes acreditados y sobre los logros de aprendizajes de los estudiantes, comparando sus desempeños antes y después de la certificación. Luego, será importante triangular esos resultados con la percepción de mejoramiento que reportan los docentes certificados en este estudio. 


\section{REFERENCIAS BIBLIOGRÁFICAS}

American Educational Research Association, American Psychological Association \& National Council on Measurement in Education. (1999). Standards for educational and psychological testing. Washington, DC: American Psychological Association.

Araya, Taut, Santelices, Manzi \& Miño (en prensa). Teoría de acción del programa de Asignación de Excelencia Pedagógica en Chile. Revista de Educación, España (ISSI), 359, Septiembrediciembre 2012. Fecha de entrada: 10-03-2010 Fecha de aceptación: 17-09-2010.

Arón, A.M. \& Milicic, N. (2000). Climas Sociales Tóxicos y Climas Nutritivos para el Desarrollo Personal en el Contexto Escolar. Revista Psykhé, Vol. 9(1), pp. 117-124.

Avalos, B. \& Assael, J. (2006). Moving from resistance to agreement: the case of the chilean teacher performance evaluation. International Journal of Educational Research. 45, 254-266.

Baker, E.L. y Linn, R.L. (2002). Validity Issues for Accountability Systems. Center for Research on Evaluation, Standards, and Student Testing (CRESST), Graduate School of Education \& Information Studies, University of California Los Angeles: Technical Report 585.

Castillo, E. \& Vásquez, L. (2003). El rigor metodológico en la investigación cualitativa. Revista Colombia Médica. 3(34), 164-167.

Cox, C. (2003). Políticas educacionales en el cambio de siglo. Santiago, Chile: Editorial Universitaria.

Darling-Hammond, L. (1999). Target time toward teachers. Journal of Staff Development. 20(2), 31-36.

Elmore, R. (2008). School Reform from the Inside Out. Cambridge, M.A.: Harvard Education Press.

Fast, E.F. y Hebbler, S., with ASR-CAS Joint Study Group on Validity in Accountability Systems (2004). A framework for examining validity in state accountability systems. Documento de la serie: Implementing the State Accountability System Requirements Under the No Child Left Behind Act of 2001 (NCLB). Washington, D.C.: Council of Chief State School Officers.

Flick, U. (2004). Introducción a la investigación cualitativa. Madrid: Ediciones Morata.

Fullan, M. (2004). Learning to lead change. Building system capacity. Core concepts. Publication produced in partnership with Microsoft's Partnership in Learning (PiL) Initiative. Extraído el 14 marzo 2011 de http: //www. michaelfullan. ca/Articles_04/CoreConcepts. pdf

Goldhaber, D. (2009). Teacher Pay Reforms: The Political Implications of Recent Research. Center for American Progress Working Paper. Extraído el 4 febrero 2010 de http: //www. americanprogress. org/issues/2006/12/pdf/teacher_pay_report. pdf

Harvey-Beavis, O. (2003). Performance-Based Rewards for Teachers: A Literature Review. Paper prepared for the Organisation for economic co-operation and development (OECD), Paris, France. Extraído el 4 febrero 2010 de http: //www. oecd. org/dataoecd/17/47/34077553. pdf

Hsieh, H.F. \& Shannon, S.E. (2005). Three approaches to qualitative content analysis. Qualitative Health Research, 9(15), 1277-1288. ttp: //unesdoc. unesco. org/images/0013/001330/133010e.pdf

Joint Committee on Standards for Educational Evaluation (1988). The personnel evaluation standards. Newbury Park, CA: Corwin Press.

Kane, M. (2002). Validating high-stakes testing programs. Educational Measurement: Issues and Practice. $\mathrm{N}^{\mathrm{o}}$ 21(1), pp. 31-41.

Lane, S. \& Stone, C.A. (2002). Strategies for examining the consequences of assessment and accountability programs. Educational Measurement: Issues and Practice. 21(1), 23-30.

Lane, S., Park, C.S., \& Stone, C.A. (1998). A framework for evaluating the consequences of assessment programs. Educational Measurement: Issues and Practice, 17(2), 24-28.

Linn, R.L. (1997). Evaluating the validity of assessments: the consequences of use. Educational Measurement: Issues and Practice, 16, 14-16. 
Manzi, J., Araya, C., González, R., Barros, E., Bravo, D., Peirano, C., Cadiz, J., y Torres, D. (2007). Validity evidence for the certification of teaching excellence in Chile: A pioneer experience in Latin America. Chicago: Documento presentado en la Reunión Anual de la Asociación Americana de Investigación en Educación (AERA) el 13 de Marzo.

Mc Kinsey \& Company, Social Sector Office. (2007). How the world's Best-Performing School Systems Come Out On Top. Disponible en sitio web: http: //www. mckinsey. com/locations/ ukireland/publications/pdf/Education_report. pdf

Mehrens, W.A. (1997). The consequences of consequential validity. Educational Measurement: Issues and Practice, 16, 16-19.

Messick, S. (1994). The interplay of evidence and consequences in the validation of performance assessments. Educational Researcher, 23(2), 13-23.

Messick, S. (1998). Test validity: a matter of consequence. Social Indicators Research, 45, 35-44.

Mineduc (2002). Decreto con fuerza de ley. Fija las normas que estructuran y organizan el funcionamiento y operación de la asignación de excelencia pedagógica y la red maestros de maestros. Ministerio de Educación de Chile. Extraído el $1^{\circ}$ febrero 2010 de http: //www. leychile. cl/N avegar?idNorma $=195372 \&$ buscar $=$ asignacion + excelencia + pedag $\% \mathrm{C} 3 \% \mathrm{~B} 3$ gica

Mineduc (2004a). La educación chilena en el cambio de siglo: políticas, resultados y desafíos. Informe Nacional de Chile, Oficina Internacional de Educación, UNESCO. Ministerio de Educación. Santiago, Chile.

Mineduc (2004b). Marco para la buena enseñanza. Ministerio de Educación. Santiago, Chile.

Mizala, A. \& Romaguera, P. (2002). Regulación, incentivos y remuneraciones de los profesores en Chile. Documento sin publicar. Documento de Trabajo $N^{o} 116$, Serie Economía, Departamento de Ingeniería Industrial, Universidad de Chile.

Moss, P.A. (1992). Shifting conceptions of validity in educational measurement: implications for performance assessment. Review of Educational Research, 62, 229-258.

Moss, P.A. (1998). The role of consequences in validity theory. Educational Measurement: Issues and Practice, 17(2), 6-12.

National Research Council (2008). Assessing accomplished teaching: advanced - level certification programs. Washington, D.C.: The National Academic Press.

Odden, A.R. \& Kelley, C.J. (2002). Paying teachers for what they know and do. (2 ${ }^{\text {nd }}$ Ed.). Thousand Oaks, CA: Corwin Press.

Patton, M.Q. (2008). Utilization - focused evaluation (4 ${ }^{\text {rd }}$ Ed.). Beverly Hills, CA: Sage.

Patton, M.Q. (2002). Qualitative research and evaluation methods ( $3^{\text {rd }}$ Ed.). Thousand Oaks, CA: Sage.

Popham, W.J. (1997). Consequential validity: right concern-wrong concept. Educational Measurement: Issues and Practice, 6, 9-14.

Sclafani, S. (2010). Teacher Compensation around the globe. Kappan. Vol. 19(8), pp. 38-43.

Shepard, L.A. (1997). Evaluating centrality of test use and consequences of test validity. Educational Measurement: Issues and Practice, 6, 71-86.

Vaillant, D. (2009). La profesión docente: lecciones para diseñadores de políticas sobre reformas que funcionan. Cap. 4 En Schwartzman, S. \& Cox, C. [Eds] Políticas educativas y cohesión social en América Latina. Santiago, Chile: Uqbar Editores.

Villegas-Reimers, E. (2003). Teacher professional development: an international review of the literature. Paris: UNESCO, Institute for Educational Planning. Disponible en sitio web: http: //unesdoc. unesco. org/images/0013/001330/133010e. pdf 